\title{
Fractionalization of the linear cyclic transforms
}

\author{
T. Alieva and M. L. Calvo \\ Departamento de Optica, Facultad de Fisicas, Universidad Complutense, 28040 Madrid, Spain
}

Received February 22, 2000; revised manuscript received June 21, 2000; accepted June 23, 2000

\begin{abstract}
In this study the general algorithm for the fractionalization of the linear cyclic integral transforms is established. It is shown that there are an infinite number of continuous fractional transforms related to a given cyclic integral transform. The main properties of the fractional transforms used in optics are considered. As an example, two different types of fractional Hartley transform are introduced, and the experimental setups for their optical implementation are proposed. (C) 2000 Optical Society of America [S0740-3232(00)00312-4] OCIS codes: $070.0070,070.2590,070.6020$.
\end{abstract}

\section{INTRODUCTION}

Recently, several methods for the fractionalization of the different types of linear transform have been proposed. ${ }^{1-9}$ The fractional transforms are now actively used in signal and image processing and optics, particularly in Fourier optics. $^{2}$ The advantage of various types of fractional transform, such as the fractional Fourier, Hankel, and Hilbert transforms,${ }^{1-6}$ is that they can be optically implemented, which enhances their usefulness in optical signal processing and image techniques, in general. These transforms are relatives of the well-known Fourier, Hankel, and Hilbert transforms widely used in optical systems for a great variety of optical operations, including correlation and filtering.

Previously, one of the possible methods for modeling the fractionalization of the cyclic transform with period $N$, based on the assumption that the fractional $R$-transform is a weighted combination of the integer order of $R$, was proposed in Refs. 7 and 8. Nevertheless, this definition is not unique. Thus there exist the fractional Fourier transforms and the fractional Hankel transforms that are defined through another procedure., ${ }^{1,6}$

The goal of this paper is to establish a general algorithm for the fractionalization of the linear cyclic integral transforms and to consider their main characteristics and fundamental properties. As an example, the different types of fractional Fourier and fractional Hartley transform are studied and analyzed.

The paper is organized as follows. In Section 2 we introduce the definition of the term linear cyclic integral transform. Section 3 is dedicated to the fractionalization procedure for cyclic transforms. The structure of the particular kernels is analyzed, and fundamental properties are demonstrated. Some specific cases are discussed. In Section 4 we apply the previous formalism (Section 3) to represent the kernel as a linear superposition of a finite number of harmonics, demonstrate the consistency of the result, and give some useful examples related to a sampling theorem. Section 5 introduces a generalization of the previous procedure (Section 4), and several examples are discussed. Section 6 is dedicated to the common existing properties of the various types of cyclic transforms; Section 7, to the case of the fractional Hartley transform.
We end the paper with a discussion and conclusions in Section 8.

\section{LINEAR CYCLIC INTEGRAL TRANSFORMS}

We start from the definition of the term cyclic transform. For simplicity we will consider the one-dimensional case. Let $R$ be an operator of a linear integral transform:

$$
R[f(x)](u)=\int K(x, u) f(x) \mathrm{d} x .
$$

A linear integral transform is a cyclic one if its $N$-time performance produces the identity transform

$$
R^{N}=I \text {. }
$$

There is a long list of well-known and widely used cyclic transforms. The Fourier and Hilbert transforms are cyclic with the period $N=4$. The Hankel and Hartley transforms have the period $N=2$. The cyclic canonical transforms of period $N$ with kernel

$$
K(x, u)=\frac{1}{\sqrt{i B}} \exp \left[i \pi\left(A x^{2}+D u^{2}-2 x u\right) / B\right],
$$

where $A+D=2 \cos (2 \pi m / N)$ and $m, N$ are integers, were studied in Ref. 10 .

All these transforms have some common properties. Thus the eigenvalues of the cyclic transforms can be represented as $A=\exp (i 2 \pi L / N)$, where $L$ is an integer. Indeed, let $\Psi(x)$ be an eigenfunction of $R$ with eigenvalue $A=a \exp (i \varphi)$, where $a$ and $\varphi$ are real and $a>0$. From Eq. (2) one obtains $A^{N}=1$, then $a=1$, and $\varphi=2 \pi L / N$.

The eigenfunction $\Psi(x)$ of such transforms with eigenvalue $A=\exp (i 2 \pi L / N)$ can be constructed from the arbitrary generator function fwe assume only that $R[g(x)]$ exists $\}$ by the following procedure:

$$
\Psi(x)=\frac{1}{N} \sum_{n=0}^{N-1} \exp (-i 2 \pi n L / N) R^{n}[g(u)](x) .
$$

Indeed, 


$$
\begin{aligned}
R[\Psi(x)](u)= & \frac{1}{N} \sum_{n=0}^{N-1} \exp (-i 2 \pi n L / N) R^{n+1}[g(x)](u) \\
= & \exp (i 2 \pi L / N) \frac{1}{N} \sum_{n=0}^{N-1} \exp [-i 2 \pi(n \\
& +1) L / N] R^{n+1}[g(x)](u) \\
= & \exp (i 2 \pi L / N) \Psi(u) .
\end{aligned}
$$

\section{FRACTIONALIZATION OF THE CYCLIC TRANSFORM}

The desirable properties of the fractional $R$-transform $R^{\alpha}$ (where $\alpha$ is a parameter of the fractionalization) are as follows:

- $R^{\alpha}$ is continuous for all real values $\alpha$;

- Additivity with respect to parameter $\alpha: R^{\alpha+\beta}$ $=R^{\alpha} R^{\beta}$

- Reproducibility of ordinary transforms for integer $\alpha$; in particular, $R^{1}=R$ and $R^{0}=R^{N}=I$.

Let us analyze the structure of the kernel of the fractional $R$-transform with period $N$. Owing to its periodicity with respect to parameter $\alpha$, one can represent $K(\alpha, x, u)$ in the form

$$
K(\alpha, x, u)=\sum_{n=-\infty}^{\infty} k_{n}(x, u) \exp \left(i 2 \pi \frac{\alpha n}{N}\right),
$$

where the coefficients $\left\{k_{n}\right\}$ have to satisfy the following system of relations:

$$
\begin{aligned}
& K(0, x, u)=\sum_{n=-\infty}^{\infty} k_{n}(x, u), \\
& K(1, x, u)=\sum_{n=-\infty}^{\infty} k_{n}(x, u) \exp \left(i 2 \pi \frac{n}{N}\right), \\
& \quad \ldots \quad \ldots \\
& K(N-1, x, u)=\sum_{n=-\infty}^{\infty} k_{n}(x, u) \exp \left[i 2 \pi \frac{(N-1) n}{N}\right] .
\end{aligned}
$$

Moreover, the coefficients have to be orthonormal to one another:

$$
\int k_{n}(x, u) k_{m}(u, y) \mathrm{d} u=\delta_{n, m} k_{n}(x, y),
$$

where $\delta_{n, m}$ denotes the Kronecker delta symbol. Indeed, as follows from the property of the additivity,

$$
\int K(\alpha, x, u) K(\beta, u, y) \mathrm{d} u=K(\alpha+\beta, x, y),
$$

and then

$$
\begin{aligned}
\sum_{n=-\infty}^{\infty} \sum_{m=-\infty}^{\infty} & \\
\times & \exp \left[i 2 \pi \frac{(\alpha n+\beta m)}{N}\right] \int k_{n}(x, u) k_{m}(u, y) \mathrm{d} u \\
& =\sum_{l=-\infty}^{\infty} k_{l}(x, y) \exp \left[i 2 \pi \frac{(\alpha+\beta) l}{N}\right],
\end{aligned}
$$

which yields the orthogonality condition (8). The coefficients $k_{n}$ could not be equal unless $k_{n}(x, u)=k_{m}(x, u)$ $=0$.

Note that all the coefficients $k_{n+m N}$ for fixed $n$ and an arbitrary integer $m$ have the same exponential factor in the system of equations (7). Therefore we can rewrite Eqs. (7) as

$$
\begin{aligned}
& K(0, x, u)=\sum_{n=-\infty}^{\infty} k_{n}(x, u)=\sum_{n=0}^{N-1} \sum_{m=-\infty}^{\infty} k_{n+m N}(x, u), \\
& K(1, x, u)=\sum_{n=0}^{N-1} \exp \left(i 2 \pi \frac{n}{N}\right) \sum_{m=-\infty}^{\infty} k_{n+m N}(x, u), \\
& K(N-1, x, u)=\sum_{n=0}^{N-1} \exp \left[i 2 \pi \frac{(N-1) n}{N}\right] \\
& \times \sum_{m=-\infty}^{\infty} k_{n+m N}(x, u) .
\end{aligned}
$$

Introducing new variables

$$
C_{n}(x, u)=\sum_{m=-\infty}^{\infty} k_{n+m N}(x, u),
$$

we obtain the system of $N$ linear equations with $N$ variables,

$$
\mathbf{B} \times \mathbf{C}=\mathbf{K} \text {, }
$$

where

$$
\begin{aligned}
& \mathbf{B}=\left[\begin{array}{ccccc}
1 & 1 & 1 & 1 & \\
1 & \exp \left(\frac{i 2 \pi}{N}\right) & \exp \left(\frac{i 4 \pi}{N}\right) & \cdots & \exp \left[\frac{i 2 \pi(N-1)}{N}\right] \\
\cdots & \cdots & \cdots & \\
1 & \exp \left[\frac{i 2 \pi(N-1)}{N}\right] & \exp \left[\frac{i 4 \pi(N-1)}{N}\right] & \cdots & \exp \left[\frac{i 2 \pi(N-1)^{2}}{N}\right]
\end{array}\right], \\
& \mathbf{C}=\left[\begin{array}{c}
C_{0}(x, u) \\
C_{1}(k, u) \\
\cdots \\
C_{N-1}(x, u)
\end{array}\right], \quad \mathbf{K}=\left[\begin{array}{c}
K(0, x, u) \\
K(1, x, u) \\
\cdots \\
K(N-1, x, u)
\end{array}\right] .
\end{aligned}
$$


This system has a unique solution because the determinant of matrix $\mathbf{B}$ is not 0 . The inverse matrix $\mathbf{B}^{-1}$ has the following form:

$\mathbf{B}^{-1}=\frac{1}{N}\left[\begin{array}{ccccc}1 & 1 & 1 & 1 \\ 1 & \exp \left[\frac{i 2 \pi(N-1)}{N}\right] & \exp \left[\frac{i 2 \pi(N-2)}{N}\right] & \cdots & \exp \left(\frac{i 2 \pi}{N}\right) \\ \cdots & \cdots & \cdots & \\ 1 & \exp \left[\frac{i 2 \pi(N-1)^{2}}{N}\right] & \exp \left[\frac{i 2 \pi(N-1)(N-2)}{N}\right] & \cdots & \left.\exp \left[\frac{i 2 \pi(N-1)}{N}\right]\right]\end{array}\right]$

and we derive the expression for the partial sum of the coefficients in the Fourier expansion (6):

$$
C_{n}(x, u)=\frac{1}{N} \sum_{l=0}^{N-1} \exp \left(-i 2 \pi \frac{l n}{N}\right) K(l, x, u) .
$$

It is easy to see that $C_{n}$ satisfy a condition similar to Eq. (8):

$$
\int C_{n}(x, u) C_{m}(u, y) \mathrm{d} u=\delta_{n, m} C_{n}(x, y) .
$$

In particular, for $N=2$ we obtain

$$
\begin{aligned}
& C_{0}(x, u)=\frac{1}{2}[K(0, x, u)+K(1, x, u)], \\
& C_{1}(x, u)=\frac{1}{2}[K(0, x, u)-K(1, x, u)],
\end{aligned}
$$

and for $N=4$ we obtain

$$
\begin{aligned}
C_{0}(x, u)= & \frac{1}{4}[K(0, x, u)+K(1, x, u)+K(2, x, u) \\
& +K(3, x, u)], \\
C_{1}(x, u)= & \frac{1}{4}[K(0, x, u)-i K(1, x, u)-K(2, x, u) \\
& +i K(3, x, u)], \\
C_{2}(x, u)= & \frac{1}{4}[K(0, x, u)-K(1, x, u)+K(2, x, u) \\
& -K(3, x, u)], \\
C_{3}(x, u)= & \frac{1}{4}[K(0, x, u)+i K(1, x, u)-K(2, x, u) \\
& -i K(3, x, u)] .
\end{aligned}
$$

Note that some partial sums for certain transforms could be equal to zero. This is the case for the Hilbert transform.

Thus, if we find the coefficients $k_{n}$ that satisfy condition (8) and whose partial sums are given by Eq. (17), we can construct the fractional transform. In general, there are several numbers of sets $\left\{k_{n}\right\}$ that generate the fractional transforms of a given $R$-transform.

\section{FRACTIONAL TRANSFORM KERNELS WITH $N$ HARMONICS}

As we have shown above, the kernel of the fractional cyclic transform can be represented as a superposition of harmonics with complex amplitudes $k_{n}$. In this section we assume that the number of harmonics is limited by $N$, where $N$ is a period of the cyclic transform. Then every sum $C_{n}(x, u)(n \in[0, N-1])$ contains only one element $k_{n+\varphi_{n}}(x, u)=C_{n}(x, u)$ from decomposition (6), where $\varphi_{n}$ $=m N$ and $m$ is an arbitrary integer. Therefore, in the general case, the kernel of the fractional $R$-transform with $N$ harmonics can be written as

$$
\begin{aligned}
K(\alpha, x, u)= & \sum_{n=0}^{N-1} k_{n+\varphi_{n}}(x, u) \exp \left[i 2 \pi \frac{\alpha\left(n+\varphi_{n}\right)}{N}\right] \\
= & \frac{1}{N} \sum_{l=0}^{N-1} K(l, x, u) \sum_{n=0}^{N-1} \exp \left(-i 2 \pi \frac{l n}{N}\right) \\
& \times \exp \left[i 2 \pi \frac{\alpha\left(n+\varphi_{n}\right)}{N}\right] .
\end{aligned}
$$

This equation provides a formula for recovering the continuous periodic function $K(\alpha, x, u)$ from its $N$ samples $K(l, x, u)$, under the assumption that the spectrum of $K(\alpha, x, u)$ contains only $N$ harmonics at the frequencies $\left\{\varphi_{0}, 1+\varphi_{1}, \ldots, n+\varphi_{n}, \ldots, N-1+\varphi_{N-1}\right\}$.

If we use $\varphi_{n}=0(n=0,1, \ldots, N-1)$, we obtain the fractional transform with the kernel

$$
\begin{aligned}
K(\alpha, x, u)= & \frac{1}{N} \sum_{l=0}^{N-1} \exp \left[i \pi \frac{(N-1)(\alpha-l)}{N}\right] \\
& \times \frac{\sin [\pi(\alpha-l)]}{\sin [\pi(\alpha-l) / N]} K(l, x, u),
\end{aligned}
$$

which was proposed by Shih in Ref. 8. In particular, this formula was used as a definition of a kind of fractional Fourier transform (for continuous and discrete cases). ${ }^{7,8}$

Choosing $N$ nonzero coefficients in decomposition (6) with indices $j=-(N-1) / 2, \ldots, 0, \ldots,(N-1) / 2$, which corresponds to $m=0$, for $n=0,1, \ldots,(N-1) / 2$, and $m$ $=-1$, for $n=(N-1) / 2+1, \ldots, N-1$, where $N$ is odd, we obtain the kernel

$$
K(\alpha, x, u)=\frac{1}{N} \sum_{l=0}^{N-1} \frac{\sin [\pi(\alpha-l)]}{\sin [\pi(\alpha-l) / N]} K(l, x, u) .
$$

This equation corresponds to the recovery procedure of the band-limited periodic function from the equidistant 
sampling. ${ }^{11}$ In particular, if $K(l, x, u)$ is real for integer $l=0,1, \ldots, N-1$, then the kernel of the fractional transform determined by Eq. (23) is real too. This also means that the Fourier spectrum of $K(\alpha, x, u)$ with respect to parameter $\alpha$ is symmetric: $\left|k_{j}\right|=\left|k_{-j}\right|$.

As an example, let us consider the general expression (21) for the kernel of the fractional $R$-transform with the period 4 (which is the case for the Fourier and the Hilbert transforms):

$$
\begin{aligned}
K(\alpha, x, u)= & \frac{1}{4} \sum_{l=0}^{3} K(l, x, u) \sum_{n=0}^{3} \exp \left(-i \pi \frac{l n}{2}\right) \\
& \times \exp \left[i \pi \frac{\alpha\left(n+\varphi_{n}\right)}{2}\right] \\
= & \frac{1}{4} \sum_{l=0}^{3} K(l, x, u) S(l),
\end{aligned}
$$

where

$$
\begin{aligned}
S(0)= & \exp \left(i \pi \frac{\alpha \varphi_{0}}{2}\right)+\exp \left[i \pi \frac{\alpha\left(1+\varphi_{1}\right)}{2}\right] \\
& +\exp \left[i \pi \frac{\alpha\left(2+\varphi_{2}\right)}{2}\right]+\exp \left[i \pi \frac{\alpha\left(3+\varphi_{3}\right)}{2}\right], \\
S(1)= & \exp \left(i \pi \frac{\alpha \varphi_{0}}{2}\right)-i \exp \left[i \pi \frac{\alpha\left(1+\varphi_{1}\right)}{2}\right] \\
& -\exp \left[i \pi \frac{\alpha\left(2+\varphi_{2}\right)}{2}\right]+i \exp \left[i \pi \frac{\alpha\left(3+\varphi_{3}\right)}{2}\right], \\
S(2)= & \exp \left(i \pi \frac{\alpha \varphi_{0}}{2}\right)-\exp \left[i \pi \frac{\alpha\left(1+\varphi_{1}\right)}{2}\right] \\
& +\exp \left[i \pi \frac{\alpha\left(2+\varphi_{2}\right)}{2}\right]-\exp \left[i \pi \frac{\alpha\left(3+\varphi_{3}\right)}{2}\right], \\
& -\exp \left[i \pi \frac{\alpha\left(2+\varphi_{2}\right)}{2}\right]-i \exp \left[i \pi \frac{\alpha\left(3+\varphi_{3}\right)}{2}\right] \\
S(3)= & \exp \left(i \pi \frac{\alpha \varphi_{0}}{2}\right)+i \exp \left[i \pi \frac{\alpha\left(1+\varphi_{1}\right)}{2}\right]
\end{aligned}
$$

Note that, for the Hilbert transform, the number of harmonics reduces to two because $C_{0}(x, u)=C_{2}(x, u)=0$, since $\quad K(0, x, u)=-K(2, x, u) \quad$ and $K(1, x, u)$ $=-K(3, x, u)$. Then the fractional Hilbert transform kernel can be written as

$$
\begin{aligned}
K(\alpha, x, u)= & \frac{1}{2} K(0, x, u)\left\{\exp \left[i \pi \frac{\alpha\left(3+\varphi_{3}\right)}{2}\right]\right. \\
& \left.+\exp \left[i \pi \frac{\alpha\left(1+\varphi_{1}\right)}{2}\right]\right\}-\frac{i}{2} K(1, x, u) \\
& \times\left\{\exp \left[i \pi \frac{\alpha\left(3+\varphi_{3}\right)}{2}\right]\right. \\
& \left.-\exp \left[i \pi \frac{\alpha\left(1+\varphi_{1}\right)}{2}\right]\right\} \\
= & \exp \left[i \pi \alpha\left(1+m_{1}+m_{3}\right)\right] \\
& \times\left\{K(0, x, u) \cos \left[\pi \alpha\left(\frac{1}{2}+m_{3}-m_{1}\right)\right]\right. \\
& \left.-K(1, x, u) \sin \left[\pi \alpha\left(\frac{1}{2}+m_{3}-m_{1}\right)\right]\right\}
\end{aligned}
$$

where $m_{1}$ and $m_{3}$ are integers. In particular, for $m_{1}$ $=m_{3}=0\left(k_{n}=0\right.$ if $\left.n \neq 1,3\right)$, one obtains

$$
\begin{aligned}
& K(\alpha, x, u) \\
& \quad=\exp (i \pi \alpha)\left[K(0, x, u) \cos \left(\frac{\pi \alpha}{2}\right)-K(1, x, u) \sin \left(\frac{\pi \alpha}{2}\right)\right],
\end{aligned}
$$

whereas, for the case in which $m_{1}=0$ and $m_{3}=-1$ $\left(k_{n}=0\right.$ if $\left.n \neq-1,1\right)$, the common form for the fractional Hilbert transform ${ }^{4}$ with a real kernel is obtained:

$$
K(\alpha, x, u)=K(0, x, u) \cos \left(\frac{\pi \alpha}{2}\right)+K(1, x, u) \sin \left(\frac{\pi \alpha}{2}\right)
$$

Therefore, even for the same number of harmonics, there are several ways to perform the fractionalization of the cyclic transforms.

\section{FRACTIONAL TRANSFORM KERNELS AND EIGENFUNCTIONS OF THE CYCLIC TRANSFORM}

In this section we consider the fractional transform kernel representation through the set of the eigenfunctions of the cyclic transform. This allows us to construct the fractional kernels with a number of harmonics $M>N$.

Assume that there is a complete set of orthonormal eigenfunctions $\left\{\Phi_{n}\right\}$ of the operator $R$ with eigenvalues $\left\{A_{n}=\exp \left(i 2 \pi L_{n} / N\right)\right\}, n=0,1, \ldots$ (see Section 2$)$ :

$$
\int \Phi_{n}(x) \Phi_{m}^{*}(x) \mathrm{d} x=\delta_{n, m} .
$$

This is the case for the Fourier and the Hartley transforms, where $\Phi_{n}(x)$ are the Hermite-Gauss modes,

$$
\Phi_{n}(x)=\left(\sqrt{\pi} 2^{n} n !\right)^{-1 / 2} \exp \left(-\frac{1}{2} x^{2}\right) H_{n}(x),
$$

and $H_{n}(u)$ are the Hermite polynomials. It is also the case for the Hankel transform of a different order, where $\Phi_{n}(x)$ are the normalized Laguerre-Gauss functions, and for many other, but not all, cyclic transforms. Thus, for 
example, the Hilbert operator $\mathscr{H}$ does not satisfy this assumption. Indeed, because of the orthogonal property of the Hilbert transform,

$$
\int_{-\infty}^{\infty} f(x) \mathscr{H}[f(u)](x) \mathrm{d} x=0,
$$

its eigenfunctions are self-orthogonal:

$$
\int_{-\infty}^{\infty} \Psi^{2}(x) \mathrm{d} x=0 .
$$

At this point one may recall that certain finite Hilbert transforms, not considered in the present case, for obvious reasons, could impose particular orthogonality conditions. $^{12}$

If the set of orthonormal eigenfunctions exists, we can represent a kernel of the $R$-transform of the integer power $p$ as

$$
\begin{aligned}
K(p, x, u) & =\sum_{n=0}^{\infty} \Phi_{n}(x)\left(A_{n}\right)^{p} \Phi_{n}^{*}(u) \\
& =\sum_{n=0}^{\infty} \Phi_{n}(x) \exp \left(i 2 \pi \frac{p L_{n}}{N}\right) \Phi_{n}^{*}(u) .
\end{aligned}
$$

Then one of the possible series of the kernels for the fractional $R$-transform can be written in the form

$$
K(\alpha, x, u)=\sum_{n=0}^{\infty} \Phi_{n}(x) \exp \left[i 2 \pi \frac{\left(L_{n}+l_{n} N\right)}{N} \alpha\right] \Phi_{n}^{*}(u),
$$

where $l_{n}$ is an integer. This kernel satisfies the additivity condition because of the orthonormality of the eigenfunctions $\Phi_{n}(x)$.

In particular, the fractional Fourier and Hankel transforms, based on this definition of fractionalization for $L_{n}$ $=-n$ and $l_{n}=0$, describe the propagation of the optical beams through a medium with a quadratic refractive index. We will call these transforms the optical fractional transforms. Each of their kernels contains an infinite number of harmonics. Thus the optical fractional Fourier transform has the following kernel:

$$
\begin{aligned}
K_{F}(\alpha, x, u)= & \sum_{n=0}^{\infty} \Phi_{n}(x) \exp \left(-i \frac{\pi n \alpha}{2}\right) \Phi_{n}^{*}(u) \\
= & \exp (i \pi \alpha / 4)[i 2 \pi \sin (\pi \alpha / 2)]^{-1 / 2} \\
& \times \exp \left[i \frac{\left(x^{2}+u^{2}\right) \cos (\pi \alpha / 2)-2 x u}{2 \sin (\pi \alpha / 2)}\right] .
\end{aligned}
$$

For $l_{n}=l=$ const., the kernel for the fractional Fourier transform is related to $K_{F}(\alpha, x, u)$ as

$$
K(\alpha, x, u)=K_{F}(\alpha, x, u) \exp (i 2 \pi l \alpha) .
$$

If we use $L_{n}=-n, l_{0}=1$, and $l_{n}=0$ for $n>0$ in Eq. (34), we obtain the following fractional Fourier transform kernel:

$$
K(\alpha, x, u)=K_{F}(\alpha, x, u)+[\exp (i 2 \pi \alpha)-1] .
$$

In general, there are an infinite number of fractional kernels, which can be constructed by use of procedure (34). The schemes of the Fourier spectra with respect to parameter $\alpha$ of the different fractional Fourier transform kernels, which indicate the nonzero coefficients $k_{n}$ in Eq (6), are given in Fig. 1. Cases (a), (b), and (c) correspond to the kernel of the optical fractional Fourier transform (35); the kernel of the fractional Shih Fourier transform (22); and the kernel (21) for $N=4$ and $\varphi_{0}=0, \varphi_{1}$ $=4, \varphi_{2}=0, \varphi_{3}=-4$, respectively.

Let us rewrite Eq. (34) in the following form:

$$
K(\alpha, x, u)=\sum_{n=-\infty}^{\infty} z_{n}(x, u) \exp \left(i 2 \pi \frac{n}{N} \alpha\right) .
$$

Here $z_{n}(x, u)$ is a sum of the elements $\Phi_{j}(x) \Phi_{j}^{*}(u)$ over $j$ where $\Phi_{j}(x)$ is the eigenfunction of the $R$-transform with the eigenvalue $\exp (i 2 \pi n / N)$. Thus, for the case of the optical fractional Fourier transform

$$
\begin{aligned}
K(\alpha, x, u) & =\sum_{n=0}^{\infty} \exp \left(-i \pi \frac{n}{2} \alpha\right) \Phi_{n}(x) \Phi_{n}^{*}(u) \\
& =\sum_{n=-\infty}^{0} z_{n}(x, u) \exp \left(i \pi \frac{n}{2} \alpha\right),
\end{aligned}
$$

the coefficients $z_{n}(x, u)=0$ for positive $n$ and $z_{n}(x, u)$ $=\Phi_{n}(x) \Phi_{n}^{*}(u)$ for $n \leqslant 0$. As we will show below, the fractional Hartley transform can be represented in the form

$$
\begin{aligned}
K(\alpha, x, u) & =\sum_{n=0}^{\infty} \exp (-i \pi \alpha n) z_{-n}(x, u), \\
z_{-n}(x, u) & =\Phi_{2 n}(x) \Phi_{2 n}(u)+\Phi_{2 n+1}(x) \Phi_{2 n+1}(u) .
\end{aligned}
$$

It is easy to see from Eq. (38) that we can generate another kernel series with $M$ harmonics,

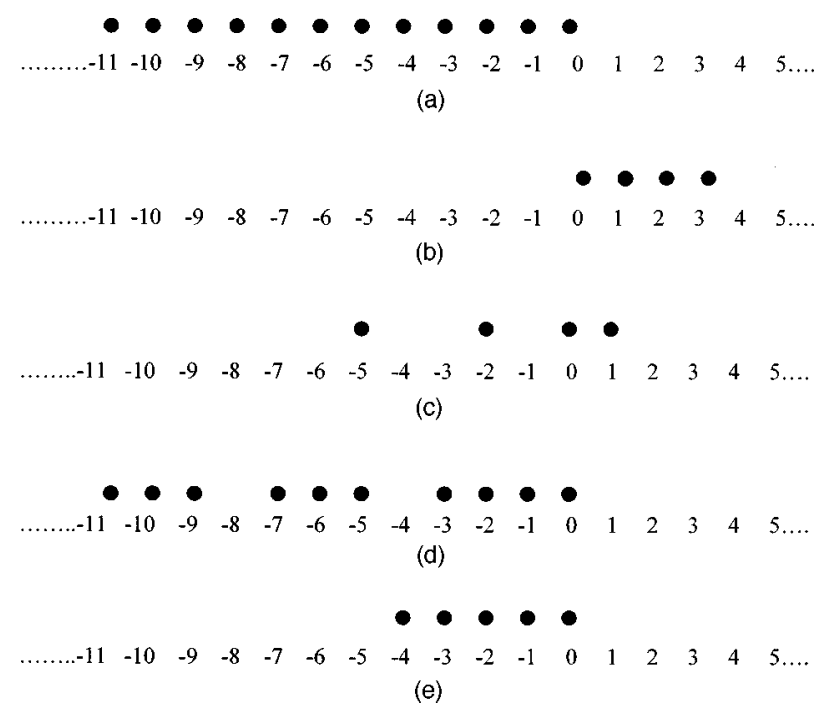

Fig. 1. Harmonic content for the different types of fractional Fourier transform, which correspond to the kernels defined by the following equations: (a) the optical fractional Fourier transform [Eq. (35)]; (b) Eq. (22) and Eq. (44) for $N=4$; (c) Eq. (21), where $N=4$ and $\varphi_{0}=0, \varphi_{1}=4, \varphi_{2}=0, \varphi_{3}=-4$; (d) Eq. (45) for $N=4$; (e) Eq. (47). 


$$
K(\alpha, x, u)=\sum_{n=0}^{M-1} \exp \left(i 2 \pi \frac{\alpha n}{M}\right) \sum_{m=-\infty}^{\infty} z_{n+M m}(x, u) .
$$

The kernels satisfy the requirements for the fractional transforms. Here the sums of the elements $z_{j}(x, u)$,

$$
k_{n}(M, x, u)=\sum_{m=-\infty}^{\infty} z_{n+M m}(x, u),
$$

are used as the coefficients $k_{n}(x, u)$ in Eq. (6). Note that Eq. (8) holds for the coefficients $k_{n}(M, x, u)$ and $k_{m}(M, x, u)$ because they are constructed from the disjoint series of the orthonormal elements. The number of harmonics $M$ has to be defined by $M=N l$, where $l$ is an integer, to satisfy to conditions (11).

One can prove that the kernel (41) for $\alpha=1$ reduces to Eq. (33). In particular, if $\left\{\Phi_{n}\right\}$ is the set of HermiteGauss modes and $z_{-n}(x, u)=\Phi_{n}(x) \Phi_{n}^{*}(u)$ for $n=0,1, \ldots$ and $z_{-n}(x, u)=0$ for negative $n$, then Eq. (41) corresponds to the series of the $M$-harmonic fractional Fourier transforms proposed in Ref. 9:

$$
\begin{aligned}
K(\alpha, x, u)= & \sum_{n=0}^{M-1} \exp \left[-i 2 \pi \frac{\alpha n(1-M)}{M}\right] \\
& \times \sum_{m=0}^{\infty} \Phi_{n+M m}(x) \Phi_{n+M m}^{*}(u) \\
= & \frac{1}{M} \sum_{n=0}^{M-1} \exp \left[i \pi \frac{(M-1)(\alpha l-n)}{M}\right] \\
& \times \frac{\sin [\pi(\alpha l-n)]}{\sin [\pi(\alpha l-n) / M]} K_{F}(n / l, x, u),
\end{aligned}
$$

where $K_{F}(n / l, x, u)$ is the kernel of the optical fractional Fourier transform. If $M=N(l=1)$, we find that the kernel of the Shih fractional transform defined by Eq. (22) (see Fig. 1(b) for $N=4$ ) can also be represented as

$$
\begin{aligned}
K(\alpha, x, u)= & \sum_{n=0}^{N-1} \exp \left[-i 2 \pi \frac{\alpha n(1-N)}{N}\right] \\
& \times \sum_{m=0}^{\infty} \Phi_{n+N m}(x) \Phi_{n+N m}^{*}(u) .
\end{aligned}
$$

In the general case some of the elements $z_{n+M m}(x, u)$ can be combined into the series, while others can be used as separate harmonic amplitudes, such as in this example:

$$
\begin{aligned}
K(\alpha, x, u)= & \sum_{m=0}^{\infty} z_{-N m}(x, u)+\sum_{n=1}^{N-1} \sum_{m=0}^{\infty} \\
& \times \exp \left[-i 2 \pi \alpha\left(\frac{n}{N}+m\right)\right] z_{-n-N m}(x, u),
\end{aligned}
$$

where the spectrum of the fractional transform kernel contains all negative harmonics except for $\mathrm{Nm}$ ( $m$ $=-1,-2, \ldots)$. The scheme of this kernel for $N=4$ is given in Fig. 1(d).

Another example is the fractional Fourier transform kernel with five harmonics,

$$
K(\alpha, x, u)=\sum_{n=0}^{4} \exp \left(-i \pi \frac{\alpha n}{2}\right) k_{-n}(x, u),
$$

whose amplitudes are

$$
\begin{aligned}
k_{0}(x, u) & =\Phi_{0}(x) \Phi_{0}^{*}(u), \\
k_{-j}(x, u) & =\sum_{m=0}^{\infty} \Phi_{j+4 m}(x) \Phi_{j+4 m}^{*}(u), \quad j=1,2,3 \\
k_{-4}(x, u) & =\sum_{m=1}^{\infty} \Phi_{4 m}(x) \Phi_{4 m}^{*}(u) .
\end{aligned}
$$

The scheme of the spectrum of this kernel is represented in Fig. 1(e).

Finally, we can conclude that, if the complete orthonormal set of eigenfunctions for a given cyclic transform exists, then an infinite number of fractional transform kernels with an arbitrary number of harmonics can be constructed.

\section{PROPERTIES OF THE FRACTIONAL TRANSFORMS}

In spite of the variety of schemes available for the construction of the fractional transforms, all of them have some common properties.

If the coefficients $k_{n}(x, u)$ in decomposition (6) are real, then the following relationship holds for the fractional transforms of a real function $f(x)$ :

$$
\left\{R_{F}^{\alpha}[f(x)](u)\right\}^{*}=R_{F}^{-\alpha}[f(x)](u) .
$$

This is the case for the optical fractional Fourier transform, the related fractional Hartley transform, and the optical fractional Hankel transform.

\section{A. Eigenfunctions of Fractional Transforms}

By analogy with Eq. (4), the eigenfunction $\Psi_{1 / M}(x)$ for the fractional transform $R^{\alpha}$ for $\alpha=1 / M$ with eigenvalue $A$ $=\exp (i 2 \pi L / M)$ can be constructed from the arbitrary generator function by the following procedure:

$$
\Psi_{1 / M}(x)=\frac{1}{M} \sum_{n=0}^{M-1} \exp (-i 2 \pi n L / M) R^{n / M}[g(u)](x) .
$$

In the limiting case $M \rightarrow \infty$ one obtains the eigenfunction for any value $\alpha$ with eigenvalue $\exp (i 2 \pi \alpha L)$ :

$$
\Psi_{\alpha}^{L}(x)=\frac{1}{N} \int_{0}^{N} \exp (-i 2 \pi \alpha L) R^{\alpha}[g(u)](x) \mathrm{d} \alpha .
$$

In particular, for fractional transforms generated by Eq. (34) (as was shown in the example for the fractional Fourier transform $\left.{ }^{13}\right)$, the functions $\Psi_{\alpha}^{L}(x)$ correspond to the 
elements of the orthogonal set $\left\{a_{L} \Phi_{L}\right\}$, where the constant factors depend on the generator function.

For the Shih definition of fractional transform (22) or (44), there are only $N$ different functions

$$
\Psi_{\alpha}^{L}(x)=a_{L} \sum_{m=0}^{\infty} \Phi_{L+m N}(x),
$$

which are self-reproducible under the fractional transformation for any $\alpha$ :

$$
R^{\alpha}\left[\Psi_{\alpha}^{L}(u)\right](x)=\exp (i 2 \pi \alpha L) \Psi_{\alpha}^{L}(x),
$$

where $a_{L}$ is a constant. The functions with different indices $L$ are orthogonal to one another.

\section{B. Complex and Real Fractional Transform Kernels}

As we have shown in Section 5, if there exists a complete orthonormal set of eigenfunctions $\left\{\Phi_{n}\right\}$ for the $R$-transform, then any coefficient in the harmonic decomposition of the fractional kernel $k_{n}(x, u)$ (6) can be expressed as a linear composition of the elements $\Phi_{j}(x) \Phi_{j}^{*}(u)$. For the kernel of the fractional transform to be real, the Fourier spectrum of the fractional kernel with respect to parameter $\alpha$ must be symmetric. This means that $\left|k_{-n}(x, u)\right|=\left|k_{n}(x, u)\right|$. Because the coefficients $k_{n}(x, u)$ with different indices $n$ contain the disjoint series of the orthogonal elements, their amplitudes cannot be equal. Then the kernel of the $R^{\alpha}$-transform cannot be real (even if the $R$-transform kernel is a real one) in the case in which there exists a complete orthonormal set of eigenfunctions $\left\{\Phi_{n}\right\}$ for the $R$-transform.

As we have shown above, the fractional Hilbert kernel can be real, because there is no complete orthonormal set of eigenfunctions for the Hilbert transform.

\section{FRACTIONAL HARTLEY TRANSFORMS}

As an example, let us consider the fractionalization of the Hartley transform. The Hartley transform of a function $f(x)$ is defined as follows:

$$
R_{H}[f(x)](u)=\int f(x) \operatorname{cas}(2 \pi x u) \mathrm{d} x,
$$

where $\operatorname{cas}(x)=\cos x+\sin x=\sqrt{2} \cos (x-\pi / 4)$. The forward and the inverse Hartley transforms are identical in form. This means that each is a cyclic transform with $N=2$. Moreover, the Hartley transform of a real $f(x)$ is real, too, which might be important for optical image processing.

The Hartley transform is closely related to the Fourier transform $R_{F}$. Thus an alternative method of defining $R_{H}$ is given by

$$
\begin{aligned}
R_{H}[f(x)](u)= & \frac{1}{\sqrt{2}}\left\{\exp (i \pi / 4) R_{F}[f(x)](u)\right. \\
& \left.+\exp (-i \pi / 4) R_{F}^{3}[f(x)](u)\right\} .
\end{aligned}
$$

The Hartley and the Fourier transforms have the same set of orthogonal eigenfunctions (Hermite-Gauss functions), but with different eigenvalues. Thus

$$
\begin{aligned}
R_{H} & {\left[\Psi_{n}(x)\right](u) } \\
& =\left\{\begin{array}{l}
\Psi_{n}(u) \quad \text { for } n=4 m, \quad n=1+4 m \\
-\Psi_{n}(u) \quad \text { for } n=2+4 m, \quad n=3+4 m
\end{array}\right.
\end{aligned}
$$

where $m$ is a nonnegative integer.

Because there exists a complete orthonormal set of eigenfunctions $\left\{\Phi_{n}\right\}$ for the Hartley transform, the kernel of the fractional Hartley transform $H(\alpha, x, u)$ cannot be real, despite the fact that the kernel of the Hartley transform is real.

One of the possible kernels of the fractional Hartley transform, which we call the optical fractional Hartley transform, can be written as

$$
\begin{aligned}
H(\alpha, x, u)= & \sum_{n=0}^{\infty} \exp (-i \pi \alpha n)\left[\Psi_{2 n}(x) \Psi_{2 n}(u)\right. \\
& \left.+\Psi_{2 n+1}(x) \Psi_{2 n+1}(u)\right]
\end{aligned}
$$

Comparing Eqs. (39) and (55), we find that the relationship between the optical fractional Hartley transform operator $R_{H}^{\alpha}$ and the optical fractional Fourier operator $R_{F}^{\alpha}$ is given by

$$
R_{H}^{\alpha}=\exp (i \pi \alpha / 4)\left[\cos (\pi \alpha / 4) R_{F}^{\alpha}-i \sin (\pi \alpha / 4) R_{F}^{\alpha+2}\right] .
$$

This is the generalization of Eq. (53) for an arbitrary parameter $\alpha$. Equation (56) shows the procedure for achieving the optical realization of this type of fractional Hartley transform, inasmuch as the fractional Fourier transform optical setup is well known. ${ }^{2}$ One of the possible schemes is given in Fig. 2.

Let us comment briefly about the optical setup used in performing the fractional Hartley transform for parameter $\alpha$. This is a Mach-Zender interferometer that has particular optical elements in the two arms.

Let $f(x)$ be an input complex field amplitude. First, we have to perform a fractional Fourier transform, $R^{\alpha}[f(x)]$, by using a lens with focal length $\mathbf{f}$ at a distance $d$ $=2 \mathbf{f} \sin ^{2}(\pi \alpha / 4)$ from partial (or intermediate) input and output planes. ${ }^{2}$ Second, this optical signal interacts with a phase plate $\phi=\pi \alpha / 4$. A beam splitter separates the two arms of the interferometer. The reflected beam (beam 1) interacts with an absorbing plate: $\cos (\pi \alpha / 4)$. The direct transmitted beam (beam 2) interacts with an optical system, producing the fractional Fourier transform for $\alpha=2$, which corresponds to the inverse transformation: $R^{2}[g(x)](u)=g(-u)$. Then, after reflection from mirror M1, beam 2 interacts with an absorbing plate: $\sin (\pi \alpha / 4)$, producing a phase shift equal to $-\pi / 2$. This beam is reflected from mirror M2 to recombine with beam 1 . We notice that this operation is a modification of that used for optical implementation of the Hartley transform. $^{14}$ One peculiarity is the use of the two absorbing plates. So we can conclude that this could be an interesting procedure to perform with the use of particular filtering devices in the fractional Hartley domain.

Another kernel for the fractional Hartley transform that contains only two harmonics $n_{1}$ and $n_{2}$, where $n_{1}$ $-n_{2}$ is an odd integer, is obtained from Eq. (21): 


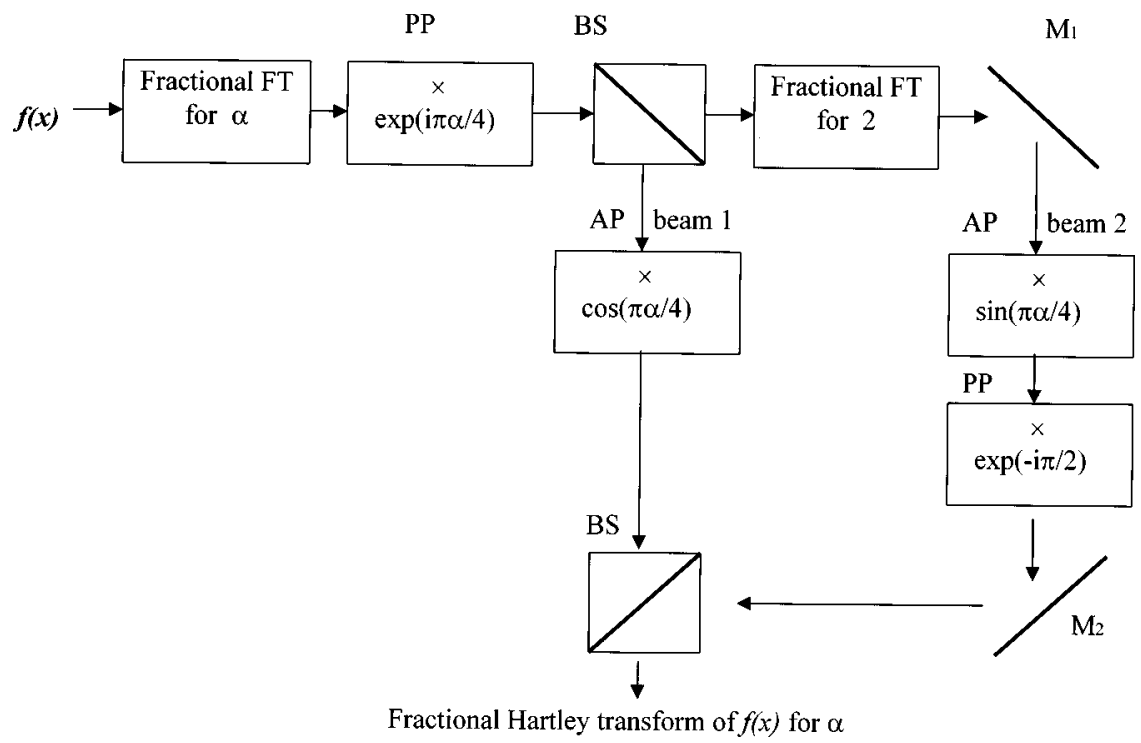

Fig. 2. Setup for the optical realization of the fractional Hartley transform defined by Eq. (56) (see text for details). PP, phase plate; $\mathrm{BS}$, beam splitter; AP, absorbing plate; M's, mirrors.

$$
\begin{aligned}
H(\alpha, x, u)= & \exp \left[\frac{i}{2} \pi \alpha\left(n_{1}+n_{2}\right)\right] \\
& \times\left\{\delta(x-u) \cos \left[\frac{\pi \alpha}{2}\left(n_{1}-n_{2}\right)\right]\right. \\
& \left.-i \operatorname{cas}(2 \pi x u) \sin \left[\frac{\pi \alpha}{2}\left(n_{1}-n_{2}\right)\right]\right\} .
\end{aligned}
$$

In particular, for $n_{1}=0$ and $n_{2}=1$, Eq. (58) is

$$
\begin{aligned}
H(\alpha, x, u)= & \exp \left(\frac{i}{2} \pi \alpha\right)\left[\delta(x-u) \cos \left(\frac{\pi \alpha}{2}\right)\right. \\
& \left.-i \operatorname{cas}(2 \pi x u) \sin \left(\frac{\pi \alpha}{2}\right)\right] .
\end{aligned}
$$

This gives another procedure for optical implementation of a particular fractional Hartley transform. In this case we should require two interferometers, one to obtain the ordinary Hartley transform $R_{H}^{1}$, as has been already done by Bracewell et al. in Ref. 14, and a second Mach-Zendertype interferometer to obtain $R_{H}^{\alpha}$.

In general, there are an infinite number of continuous fractional Hartley transforms.

\section{CONCLUSIONS}

In this paper we have established a general method for the generation of the different types of fractional transforms for a given cyclic transform. The usefulness of a specific transform is related to its feasibility in hybrid, optical, or numerical operations as well as to its applications in signal or image processing. We have proved that the analysis of harmonic contents for various types of fractional Fourier transform offers a procedure for their experimental realization. Also, we have proposed a particular experimental device based on optical correlation for the optical implementation of the fractional Hartley transform. This seems to be a new optical operation in- asmuch as it is a modification of the optical Hartley transform for performance of particular filtering procedures.

Furthermore, the fractional Fourier and Hankel transforms generated through procedure (34) have attracted the attention of the optical community because they describe in the paraxial approximation the beam propagation through a quadratic refractive-index medium. Moreover, the fractional Fourier transform is used for the development of new filtering devices. ${ }^{3}$

The fractional Hilbert transform generated by Eq. (28), which also can be optically implemented, is used for edge detection. ${ }^{5}$ The authors are currently developing further results, not discussed here, for optical applications of particular fractional transforms.

Although in this paper we have considered the cyclic integral transforms, the main results are also valid for discrete cyclic transforms.

\section{ACKNOWLEDGMENTS}

This research was financially supported by the Rectorate of the Complutense University of Madrid (UCM), under Multidisciplinary Project PR486/97-7477/97. T. Alieva is grateful for a grant from UCM. Partial results of this study were presented at the International Conference on Optical Science and Applications for Sustainable Development, Dakar (Senegal), April 10-14, 2000.

The authors e-mail addresses are talieva @eucmos.sim.ucm.es and mlcalvo@eucmax.sim.ucm.es.

\section{REFERENCES}

1. D. Mendlovic and H. M. Ozaktas, "Fractional Fourier transforms and their optical implementation," J. Opt. Soc. Am. A 10, 1875-1881 (1993).

2. A. W. Lohmann, D. Mendlovic, and Z. Zalevsky, "Fractional transformation in optics," in Progress in Optics, XXXVIII, E. Wolf, ed. (Elsevier, Amsterdam, 1998), pp. 263-342.

3. H. M. Ozaktas, M. A. Kutay, and D. Mendlovic, "Introduc- 
tion to the fractional Fourier transform and its applications," Adv. Imaging Electron Phys. 106, 239-291 (1999).

4. A. W. Lohmann, D. Mendlovic, and Z. Zalevsky, "Fractional Hilbert transform," Opt. Lett. 21, 281-283 (1996).

5. A. W. Lohmann, E. Tepichin, and J. G. Ramirez, "Optical implementation of the fractional Hilbert transform for twodimensional objects," Appl. Opt. 36, 6620-6626 (1997).

6. L. Yu, Y. Lu, X. Zeng, M. Huang, M. Chen, W. Huang, and $\mathrm{Z}$. Zhu, "Deriving the integral representation of a fractional Hankel transform from a fractional Fourier transform," Opt. Lett. 23, 1158-1160 (1998).

7. B. W. Dickinson and K. Steiglitz, "Eigenvectors and functions of the discrete Fourier transform," IEEE Trans. Acoust. Speech Signal Process. 30, 25-31 (1982).

8. C. C. Shih, "Fractionalization of Fourier transform," Opt. Commun. 118, 495-498 (1995).
9. S. Liu, J. Jiang, Y. Zhang, and J. Zhang, "Generalized fractional Fourier transforms," J. Phys. A 30, 973-981 (1997).

10. T. Alieva and M. J. Bastiaans, "Powers of transfer matrices determined by means of eigenfunctions," J. Opt. Soc. Am. A 16, 2413-2418 (1999)

11. D. A. Linden, "A discussion of sampling theorems," Proc. IRE 47, 1219-1226 (1959).

12. R. P. Kanwal, Linear Integral Equations: Theory and Techniques (Academic, New York, 1971), Chaps. 8 and 9.

13. T. Alieva and A. Barbe, "Self-fractional Fourier functions and selection of modes," J. Phys. A 30, L211-L215 (1997).

14. R. N. Bracewell, H. Bartelt, A. W. Lohmann, and N. Streibl, "Optical synthesis of the Hartley transform," Appl. Opt. 24, 1401-1402 (1985) 